American Journal of Pharmaceutical Education 2019; 83 (1) Article 6432.

\title{
RESEARCH
}

\section{Impact of Diabetes Simulation on Empathy in Pharmacy Students}

\author{
Debra Parker, PharmD, Atem Fontem, PharmD, Etabphoh Ojong, Janelle Pope, PharmD \\ University of Findlay College of Pharmacy, Findlay, Ohio \\ Submitted March 29, 2017; accepted August 23, 2017; published February 2019.
}

Objective. To assess the impact of a diabetes simulation activity on empathy in pharmacy students. Methods. Students enrolled in an elective diabetes course were required to complete a 3-day diabetes simulation. Pre- and post-activity survey questions were administered to assess the effect of the simulation activity on empathy. Students maintained daily logs of their actual blood glucose readings (tested twice a day), pre-meal simulated blood glucose readings, and simulated insulin doses. Students were required to take a placebo oral medication and to respond (and document their approach) to a simulated hypo- or hyperglycemic event.

Results. The pre- and post-activity survey results indicated statistically significant changes in responses regarding empathy.

Conclusion. This 3-day diabetes simulation increased student empathy and confidence in managing select aspects of diabetes.

Keywords: empathy, pharmacy, student, simulation, diabetes

\section{INTRODUCTION}

Empathy is an essential component in the development of caring, therapeutic relationships with patients. An empathic health care professional is one who correctly acknowledges the emotional state of a patient without experiencing the state itself. ${ }^{1}$ Empathy for patients is an important quality to develop and can be a challenging quality to objectively assess in developing health care professionals. One way in which this can be achieved is through simulation. Simulation is a bridge between the classroom learning and active real-life clinical experiences. This learning process involves role play with actors as patients attempting to create a realistic interaction to enhance medical knowledge. It is a shift from the traditional instructorcentered method. It assists learners in creating a customized experience of real-life situations, and assists students in gaining powerful insights to the consequences of their actions.

Diabetes is a chronic condition that requires continuous medical care and education to prevent acute problems and reduce the risk of long-term complications; as such, it can be very demanding for patients to adhere to medication, dietary and other lifestyle modifications to optimally control their condition. ${ }^{2}$ The required, traditional diabetes curriculum at the University of Findlay College of Pharmacy consists of lectures, discussions,

Corresponding Author: Debra Parker, 1000 N. Main St., Findlay, OH, 45840. Tel: 419-434-4450. E-mail:

parker@findlay.edu and laboratory sessions to provide students with background knowledge and basic diabetes management skills. Such activities, however, do not allow students to develop a sense of the challenges patients with diabetes face. Therefore, students do not necessarily develop an appreciation or an understanding of the challenges their patients face in their day-to-day lives. Consequently, students may not be able to develop a sense of empathy as they provide diabetes management and education.

To increase comfort level with various daily tasks that patients with diabetes are often asked to perform, as well as to assist students in developing a sense of empathy, a 3-day diabetes simulation activity was incorporated as a component of an elective diabetes course at the University of Findlay. Pre- and post-activity survey questions were given to assess changes in the students' empathy and confidence in managing patients with diabetes. Similar articles have been previously published describing diabetes simulation activities in pharmacy and other health profession students. These articles, however, were qualitative descriptions only, and did not include statistical analysis of the impact of the academic exercises. ${ }^{3-10}$ Accordingly, this study provides an analysis of pre- and post-simulation student attitudes and confidence levels in their ability to provide care for patients with diabetes.

\section{METHODS}

This study consisted of a retrospective review of a classroom survey. It was reviewed and deemed exempt by the University of Findlay Institutional Review Board. 


\section{American Journal of Pharmaceutical Education 2019; 83 (1) Article 6432.}

Data from an elective course that met 2 hours weekly for 15 weeks in the spring of 2014, 2015 and 2016 were analyzed. In this course, pharmacy students at the University of Findlay in their second or third professional year participated in a diabetes simulation activity designed to develop confidence in diabetes management and increase empathy toward future patients. The material presented throughout the course included instruction on evidencebased treatment guidelines and medications used to control and treat type 1, type 2 and gestational diabetes. It also included practical treatment of hypoglycemia and correction of hyperglycemia for patients taking insulin. The simulation activity consisted of having students simulate being a diabetic patient for 3 days. Students were required to maintain a daily log that included: actual blood glucose readings (students were asked to perform self-blood glucose monitoring twice daily), pre-meal simulated blood glucose readings (generated randomly online and accessed by the student immediately prior to eating), projected required insulin doses for each simulated blood glucose as well as for all carbohydrates consumed throughout the day as meals and snacks (calculated by the student based on an insulin: carbohydrate ratio and an insulin sensitivity factor provided at the beginning of the simulation). Students were asked to inject normal saline as a mock insulin dose once during the simulation. They also were required to take a placebo "oral medication" twice daily. Black jelly beans were used, as they were deemed as something most students would not be enthusiastic about taking twice daily.

For the testing of actual blood glucose twice daily, students were able to choose any two of the following testing times: before the first meal of the day (fasting), before and after any meal, before and after exercise, and at bedtime. For the testing and recording of the simulated blood glucose readings, students were provided with a link on their classroom website that would generate a random blood glucose within parameters pre-specified by the instructor. At the end of each day, the student had a total of four readings: two real and two simulated readings. Should the student have a snack, he or she was to assume the pre-snack reading was $153 \mathrm{mg} /$ dL (randomly designated by the instructor). Students calculated their insulin bolus doses based on their pre-meal simulated blood glucose readings and the grams of carbohydrates estimated to be in the meal or snack. For males, an insulin carbohydrate ratio of 1:8 and for females a ratio of $1: 7$ was assigned. The target pre-meal blood glucose for all participants was set at $110 \mathrm{mg} / \mathrm{dL}$.

The insulin correction factor was pre-specified as $35 \mathrm{mg} / \mathrm{dL} / 1$ unit insulin for male students and $55 \mathrm{mg} / \mathrm{dL} /$ 1 unit insulin for female students. During the 3-day simulation, each student received at least one text message from the instructor at a randomly selected time for the student, advising him or her to treat a mock hypo- or hyperglycemic event that needed immediate attention. Students were expected to calculate the correct dose of insulin in a hyperglycemic event and text the instructor back with the correct answer. In a hypoglycemic event, students were expected to text the instructor providing details on how they treated this event.

Prior to, and immediately following, the simulation activity, students completed the same validated survey (Table 1). To allow for anonymity as well as a comparison of any changes in responses, students were asked to place a 4-digit code known only to each student at the top of their pre-activity and post-activity surveys so their responses could be paired. In this survey, students were asked to respond to 16 questions that assessed either confidence or empathy. Possible answers were based on a Likert scale, with $1=$ strongly disagree, $2=$ disagree, $3=$ agree, and $4=$ strongly agree.(Figure 1). Once the post-activity survey was completed and submitted, an instructor-facilitated, inclass discussion and reflection was held. Participation in the activity was an expectation of the course, but no grade was assigned to students related to the simulation.

A Wilcoxon signed-rank test was used to analyze survey responses, with an alpha level of significance set at .05. Pre- and post-survey scores were compared for each question, with each student serving as his or her own control. The difference between each student's post-survey and pre-survey response for each question was determined, and was categorized as positive if the number chosen on the post-survey was higher than the pre-survey, and negative if the number was lower, or unchanged on the post-survey.

\section{RESULTS}

Survey data for 50 students were included for analysis, including anonymous identifiers to allow pre- and post-simulation survey results to be paired. This accounted for $100 \%$ of students who took this course during the specified time period. One student was missing a response to question 1 on the post-survey. Questions 1-6 were designed to assess empathy. A more "positive" response for questions 1-4 correlated with increased empathy. A more "negative" response for questions 5 and 6 correlated with increased empathy. Questions 7-16 were designed to assess students' self-confidence in diabetes management. A more positive response correlated with increased self-confidence. For questions 1, 3, and 4, pre- and post-survey results were not statistically significant. The changes in responses to questions $2(p=.03)$ and 5-16 $(p<.001)$ were statistically significant; responses were more positive on the postsurvey as compared to the pre-survey for questions 2 and 7-16. For questions 5 and 6, more students responded 


\section{American Journal of Pharmaceutical Education 2019; 83 (1) Article 6432.}

Table 1. Diabetes Simulation Survey Questions Posed to Student Pre- and Post-Simulation

\begin{tabular}{ll}
\hline Number & \multicolumn{1}{c}{ Question } \\
\hline 1 & I believe that having Type 1 diabetes would be difficult. \\
2 & I believe that having Type 2 diabetes would be difficult. \\
3 & I believe that it would be difficult to adjust my current lifestyle if I had diabetes. \\
4 & I believe that patients with Type 2 diabetes deserve to have the disease. \\
5 & I believe there is NO excuse for a diabetic patient to miss a dose of medication. \\
6 & I believe there is NO excuse for a diabetic patient to NOT follow a strict diet and exercise routine. \\
7 & I feel confident in my ability to administer insulin. \\
8 & I feel confident in my ability to teach someone else how to administer insulin. \\
9 & I feel confident in my ability to check blood glucoses with a home meter. \\
10 & I feel confident in my ability to teach someone else how to check blood glucoses with a home meter. \\
11 & I feel confident in my ability to count carbohydrates in my meals. \\
12 & I feel confident in my ability to teach someone else how to count carbohydrates in their meals. \\
13 & I feel confident in my ability to adjust insulin doses based on the carbohydrate content of my meals. \\
14 & I feel confident in my ability to teach someone else how to adjust insulin doses based on the carbohydrate \\
15 & content of their meals. \\
16 & I feel confident in my ability to adjust insulin doses based on glucose values. \\
\hline
\end{tabular}

negatively to the statement, which indicates increased empathy due to the way these two questions were worded (Table 2).

\section{DISCUSSION}

As hypothesized, the simulation activity positively affected student empathy toward patients with diabetes. Student confidence in managing select aspects of diabetes management also increased as evidenced by post-survey responses. Of note, students participated in this activity as part of an elective course. Each of them had previously covered materials related to diabetes in a required course. Perceptions and beliefs formulated prior to this course may account for the fact that responses to questions 1,3 and 4 did not significantly change following this simulation exercise. Also of interest, student perceptions regarding type 2 diabetes changed significantly (question 2) while perceptions regarding type 1 diabetes did not. This may reflect that students believed from the start that type 1 diabetes would be challenging to manage and this exercise confirmed that belief. However, these same students may have underestimated how challenging it would be to manage type 2 diabetes, and these perceptions did significantly change.

Several limitations to this study should be acknowledged. Responses on the survey were self-reported as well as the completion of the simulation activity. Therefore changes in empathy and confidence in diabetes management cannot directly be correlated to completion of every component of the activity; furthermore, student reports in their daily logs could not be confirmed. Finally, to maintain anonymity in the student surveys, demographic information was excluded. This, as well as a relatively small number of participants, precluded subgroup analysis, which may be a topic of research in the future.

Simulation activities compared to case-based scenarios provide a more realistic experience and may help students better prepare for future clinical experiences. Here, students had a chance to "be the patient" and

Table 2. Diabetes Simulation: Pre- and Post-Survey Activity Student Responses $(\mathrm{N}=50)$

\begin{tabular}{lccccc}
\hline Question & Negative & Positive & Unchanged & Zstat & $\boldsymbol{p}$ value \\
\hline 1 & 2 & 7 & 40 & 1.67 & .1 \\
2 & 4 & 13 & 33 & 2.18 & .03 \\
3 & 6 & 10 & 34 & 0.88 & .38 \\
4 & 7 & 9 & 34 & 0.54 & .59 \\
5 & 21 & 2 & 27 & -3.85 & $<.001$ \\
6 & 23 & 3 & 24 & -3.80 & $<.001$ \\
7 & 3 & 30 & 17 & 4.54 & $<.001$ \\
8 & 2 & 22 & 26 & 3.97 & $<.001$ \\
9 & 3 & 19 & 28 & 3.41 & $<.001$ \\
10 & 1 & 18 & 31 & 3.90 & $<.001$ \\
11 & 1 & 26 & 23 & 4.79 & $<.001$ \\
12 & 3 & 27 & 20 & 4.42 & $<.001$ \\
13 & 2 & 32 & 16 & 5.12 & $<.001$ \\
14 & 3 & 28 & 19 & 4.59 & $<.001$ \\
15 & 1 & 26 & 23 & 4.65 & $<.001$ \\
16 & 1 & 28 & 21 & 4.83 & $<.001$ \\
\hline
\end{tabular}

Negative $=$ number of respondents whose response in post-survey declined compared to pre-survey response. Positive $=$ number of respondents whose response in post-survey increased compared to pre-survey response. Unchanged $=$ number of respondents whose post-survey and pre-survey response was unchanged Questions 1 to 6 assessed empathy and questions 7 to 16 assessed confidence 


\section{American Journal of Pharmaceutical Education 2019; 83 (1) Article 6432.}

experience the barriers that can interfere with medication and lifestyle adherence. This diabetes elective course, including the simulation activity, continues to be offered at the university, as it has been shown to improve empathy in its future health care professionals. In the future, the class may be offered to other health care students at the university to increase professional diversity and sample size. Future studies could compare empathy among students in this course who participated in the simulation activity versus students who did not.

\section{CONCLUSION}

This 3-day diabetes simulation exercise designed to assist students in developing empathy and to increase confidence in managing select aspects of diabetes resulted in statistically significant results in each of these areas. This study differs from past, similar publications in that a validated pre- and post- simulation activity survey was used to determine the changes in perceptions. Despite its limitations, this exercise may lead to a long-lasting behavioral modification on the part of the students.

\section{ACKNOWLEDGMENTS}

The authors of this study gratefully acknowledge Padmini Sekar, MS, for statistical analysis of the results.

\section{REFERENCES}

1. Halpern J. What is clinical empathy? J Gen Int Med. 2003; 18(8):670-674.

2. Diabetes Care 2017;40(Suppl 1):S1-S2 DOI: 10.2337/dc17-S001. 3. DelPrete A, Giordano C, Castiglioni A, Hernandez C. Medical students' attitudes toward non-adherent patients before and after a simulated patient-role activity and small-group discussion: revisited. Cureus. 2016;8(4):e576. doi:10.7759/cureus.576.

4. Donegan D, Mader R, Weigel S, Kennel KA. Newly diagnosed type 1 diabetes mellitus: a resident simulation. MedEdPORTAL. 2013;9:9345. http://dx.doi.org/10.15766/mep_2374-8265.9345.

5. Kerr JL, Stahnke AM, Behnen EM. Assessing empathy and selfefficacy levels of pharmacy students in an elective diabetes management course. Am J Pharm Educ. 2015;79(3):Article 42. doi:10.5688/ajpe79342.

6. Quince TA, Kinnersley P, Hales J, et al. Empathy among undergraduate medical students: a multi-centre cross-sectional comparison of students beginning and approaching the end of their course. BMC Med Educ. 2016;16:92.

7. Phillips C, Shrader S, Delea D. A week-long diabetes simulation for pharmacy students. Am J Pharm Educ. 2010;74(7):1-7.

8. Westberg SM, Bumgardner MA, Brown MC, Frueh J. Impact of an elective diabetes course on student pharmacists' skills and attitudes. Am J Pharm Educ. 2010;74(3):Article 49.

9. Whitley HP. Active-learning diabetes simulation in an advanced pharmacy practice experience to develop patient empathy. Am J Pharm Educ. 2012;76(10):Article 203.

10. Wilson SE, Prescott J, Becket G. Empathy levels in first- and third-year students in health and non-health disciplines. Am J Pharm Educ. 2012;76(2):Article 24. 